\title{
Reactive oxygen species-mediated therapeutic response and resistance in glioblastoma
}

\author{
E Singer $^{1}$, J Judkins ${ }^{1}$, N Salomonis ${ }^{2}$, L Matlaf ${ }^{1}$, P Soteropoulos ${ }^{3}$, S McAllister ${ }^{\star, 1}$ and L Soroceanu ${ }^{\star, 1}$
}

Glioblastoma (GBM) resistance to therapy is the most common cause of tumor recurrence, which is ultimately fatal in $90 \%$ of the patients 5 years after initial diagnosis. A sub-population of tumor cells with stem-like properties, glioma stem cells (GSCs), is specifically endowed to resist or adapt to the standard therapies, leading to therapeutic resistance. Several anticancer agents, collectively termed redox therapeutics, act by increasing intracellular levels of reactive oxygen species (ROS). In this study, we investigated mechanisms underlying GSC response and resistance to cannabidiol (CBD), a non-toxic, non-psychoactive cannabinoid and redox modulator. Using primary GSCs, we showed that CBD induced a robust increase in ROS, which led to the inhibition of cell survival, phosphorylated (p)-AKT, self-renewal and a significant increase in the survival of GSC-bearing mice. Inhibition of self-renewal was mediated by the activation of the p-p38 pathway and downregulation of key stem cell regulators Sox2, Id1 and p-STAT3. Following CBD treatment, a subset of GSC successfully adapted, leading to tumor regrowth. Microarray, Taqman and functional assays revealed that therapeutic resistance was mediated by enhanced expression of the antioxidant response system Xc catalytic subunit XCT (SLC7A11 (solute carrier family 7 (anionic amino-acid transporter light chain), member 11)) and ROS-dependent upregulation of mesenchymal (MES) markers with concomitant downregulation of proneural (PN) markers, also known as PN-MES transition. This 'reprogramming' of GSCs occurred in culture and in vivo and was partially due to activation of the NFE2L2 (NRF2 (nuclear factor, erythroid 2-like)) transcriptional network. Using genetic knockdown and pharmacological inhibitors of SLC7A11, we demonstrated that combining CBD treatment with the inhibition of system Xc resulted in synergistic ROS increase leading to robust antitumor effects, that is, decreased GSC survival, self-renewal, and invasion. Our investigation provides novel mechanistic insights into the antitumor activity of redox therapeutics and suggests that combinatorial approaches using small molecule modulators of ROS offer therapeutic benefits in GBM.

Cell Death and Disease (2015) 6, e1601; doi:10.1038/cddis.2014.566; published online 15 January 2015

Glioblastoma (GBM) is the most common primary brain tumor in adults and poses significant therapeutic challenges. Recent transcriptome profiling of GBM tissues yielded molecular subclasses driven by specific genetic alterations and which correlated with patient outcome. ${ }^{1-4}$ Among the four GBM subtypes (classical, neural, proneural (PN), and mesenchymal (MES)), MES identity is the hallmark of glioma aggressiveness and strongly associated with the poor outcome of patients. ${ }^{5}$ In fact, upon disease recurrence, a therapy-induced PN-MES transition (PMT) of GBM tumors has been documented in some patient samples. ${ }^{5}$ PMT may represent for GBM the equivalent of epithelial-MES transition associated with other aggressive cancers; however, the molecular mechanisms underlying this transition remain elusive. ${ }^{6} \mathrm{~A}$ subset of GBM cells with stem-like characteristics, termed glioma stem cells (GSCs), have been shown to underlie the therapeutic resistance and tumor recurrence in GBM. ${ }^{6,7}$ Uncovering the mechanisms underlying the therapeutic response and resistance of GSCs is of critical importance.
Reactive oxygen species (ROS) are natural by-products of aerobic metabolism and they can promote normal cell proliferation through the activation of growth-related signaling pathways. ${ }^{8}$ Most anticancer drugs kill their target cells, at least in part, through the generation of elevated amounts of intracellular ROS. ${ }^{9}$ ROS can exert different effects according to the basal metabolic rate of the cell. The high basal metabolic rate of cancer cells makes them more susceptible to redoxdirected therapeutics in comparison with non-transformed cells. ${ }^{10}$ Redox-directed therapeutics have been developed to act as direct inhibitors of cancer and to sensitize tumors to firstline agents; however, they are associated with significant toxicity. ${ }^{9}$ The discovery of non-toxic molecules that selectively upregulate ROS in malignant cells would be beneficial.

Cannabidiol (CBD) is a non-toxic and non-psychoactive cannabinoid that has been shown to have antitumor activity in multiple cancer types. ${ }^{11}$ Activation of $\mathrm{CB}_{1}$ and $\mathrm{CB}_{2}$ receptors has been previously shown to lead to the inhibition of tumor progression; ${ }^{12}$ however, CBD does not interact efficiently with

\footnotetext{
${ }^{1}$ California Pacific Medical Center Research Institute, San Francisco, CA, USA; ${ }^{2}$ Cincinnati Children's Hospital Medical Center, Cincinnati, OH, USA and ${ }^{3}$ Center for Applied Genomics, UMDNJ, Newark, NJ, USA

*Corresponding author: S McAllister or L Soroceanu, California Pacific Medical Center Research Institute, 475 Brannan Street, Suite 220, San Francisco, CA 94107, USA. Tel: +1 415600 1770; Fax: +1 415600 1725; E-mail: McAllis@cpmcri.org (SM) or sorocel@cpmcri.org (LS)

Abbreviations: ARE, antioxidant response element; BLI, biolouminescence imaging; CBD, cannabidiol; $\mathrm{Cl}$, combination index; $\mathrm{DCF}, 2^{\prime}, 7^{\prime}$ dichlorofluorescein diacetate; ELDA, extreme limited dilution assay; ERA, erastin; Fer, ferrostatin; GBM, glioblastoma; GSC, glioma stem cell; GSH, glutathione (reduced); IC ${ }_{50}, 50 \%$ inhibitory concentration; IHC, immunohistochemistry; MES, mesenchymal; NRF2, nuclear factor, erythroid 2-like; PMT, proneural-mesenchymal transition; PN, proneural; PTGS2, prostaglandin-endoperoxidase synthase 2; ROS, reactive oxygen species; SAS, sulfasalazine; SLC7A11, solute carrier family 7 (anionic amino-acid transporter light chain), member 11; siRNA, small interfering RNA; shRNA, short hairpin RNA; VitE, vitamin $E$

Received 04.11.14; accepted 27.11.14; Edited by A Finazzi-Agrò
} 
$\mathrm{CB}_{1}$ and $\mathrm{CB}_{2}$ receptors, and the initial site $\mathrm{CBD}$ interacts with to produce antitumor activity is unknown. Our recent study demonstrated CBD-produced robust antitumor activity against a human-derived GBM in an intracranial xenograft model; ${ }^{13}$ however, no investigations to date have interrogated the therapeutic effects of CBD on GSCs.

One of the major systems used by both normal and cancerous cells to counteract oxidative insult is the NRF2 (also known as NFE2L2) transcriptionally regulated program. ${ }^{9}$ The role of NRF2 transcriptional regulator and SLC7A11 (solute carrier family 7 (anionic amino-acid transporter light chain), member 11) in mediating GBM response and resistance to redox-directed therapeutics has not been evaluated.

In the current study, we systematically interrogated therapeutic response to CBD using several GSC lines in culture and in vivo. Our results demonstrate that while CBD exhibits significant antitumor activity, a subset of GSCs adapt by activating an extended antioxidant cellular response. We show that cotargeting GSCs using ROS modulators (CBD) and inhibitors of the antioxidant response genes together is more effective than either approach alone in halting GBM growth.

\section{Results}

CBD inhibits GSC viability in an ROS-dependent manner. Primary GSC lines 3832 and 387 were treated with a range of CBD concentrations and evaluated in viability assays. CBD inhibited the viability of 3832 and 387 GSCs with an $\mathrm{IC}_{50}$ (50\% inhibitory concentration) value of $3.5 \mu \mathrm{M}(3.4-3.6)$ and $2.6 \mu \mathrm{M}(2.5-2.7)$, respectively (Figures 1a and b). In both GSC cultures, CBD increased the production of ROS (Figures 1c and d), which was reversed in the presence of vitamin $\mathrm{E}$ (VitE) (or $a$-tocopherol). In agreement with these data, the effects of CBD on cell viability were reversed in the presence of the ROS scavenger (Figures 1e and f).

CBD treatment improves survival of mice bearing intracranial GSC xenografts. We next performed efficacy studies in vivo, using two models of intracranial GBM xenografts, established by a low number $\left(5 \times 10^{3}\right)$ of GSC lines 3832 and 387. As demonstrated in Figure 2, this low number of cells can seed highly aggressive tumors. Tumor progression is rapid and the median survival in 3832 and 387 cells was 27 and 21 days, respectively. CBD treatment significantly prolonged survival in tumor bearing mice (Figures 2a and b). Immunohistochemical (IHC) analyses of GBM xenograft tissues demonstrated that CBD treatment inhibited p-AKT, Ki67 and stimulated the activation of caspase-3 in GBM in vivo (Figure 2c). Control antibody and hematoxylin and eosin staining are shown in Supplementary Figure 2. Using bioluminescence measurements, we monitored tumor growth and response to CBD therapy in real time. Our data demonstrate that following initial inhibition of tumor growth by CBD (day 22), intracranial GBM tumors appear to resume a more rapid growth rate in spite of continuous CBD administration (Figure 2d). These data suggest that in vivo, a sub-population of CBD-treated GSCs adapted during therapy and became resistant. We next set out to investigate mechanisms underlying both response and resistance to CBD treatment in tumors derived from GSCs.

CBD inhibits GSC self-renewal in an ROS-dependent manner. We previously showed that CBD inhibits Id1 expression in primary-derived GBM lines and that Id1 genetic knockdown inhibits self-renewal and Sox2 expression in primary GBM cells. ${ }^{14}$ In this study, we directly investigated the effects of CBD on self-renewal and stemness of GSC. Using sphere formation assays (10, 100 and 1000 cells per well), we determined that CBD inhibits GSC self-renewal in both 3832 and 387 lines and this effect was partially reversed by cotreatment with the antioxidant VitE $(40 \mu \mathrm{M})$ (Figures 3a and $b$ ). Using the limited dilution tumorsphere formation assay in conjunction with extreme limiting dilution analysis (ELDA) software analysis package (available from Walter+Eliza Hall Institute, Parkville, VIC, Australia), ${ }^{15}$ we determined that the cancer stem cell frequency was significantly inhibited by CBD in both GSC lines (Figure 3b, lower panel). Mechanistically, we found that CBD inhibits the expression of Sox2, Id1, p-STAT3 and upregulates phosphorylated ( $p)$-p38 MAPK, all of which have been connected to inhibition of self-renewal and stemness in GBM. $^{16}$ Importantly, these effects were also dependent on ROS production, as demonstrated by partial or complete reversal induced by cotreatment with VitE (Figure 3c). IHC analyses of GBM xenograft tissues demonstrated that CBD treatment inhibited both Id1 and Sox2 expression in vivo (Figure 3d).

CBD treatment upregulates antioxidant response genes and induces a shift to an MES molecular phenotype. To investigate the mechanism underlying the resistant GBM phenotype suggested by partial therapeutic response (Figures 2c and d), we profiled RNA extracted from three GSC lines treated with vehicle and CBD on Affymetrix Gene St 1 DNA Arrays (Affymetrix, Inc., Santa Clara, CA, USA) (Figure 4a). Raw data is available at http://www.ncbi.nlm.nih. gov/geo/query/acc.cgi?acc=GSE57978.

Using standard analyses of microarray in conjunction with Altanalyze software (developed by Dr. Nathan Salomonis; http://www.genmapp.org/AltAnalyze/), we determined that significantly altered transcripts belong to several functional classes. Specifically, we measured a downregulation of stemness markers (MELK, OLIG2), PN markers (DLL3, PDGFRA) and proliferation markers (Ki67, Top2A) (Figure 4a). Concomitantly, we measured significant upregulation of several antioxidant response gene products (SLC7A11, NRF2) as well as MES GBM markers (CD44, TNSFR10, CEBPB; Figure 4a). Taqman validation for a subset of 12 genes was performed using additional GSC lines, as well as acutely dissociated patient GBM cells treated with CBD. Taqman analysis confirmed the molecular signature shift induced by CBD (Figure 4b). Western blot analysis further corroborated these findings in both 3832 and 387 GSC lines (Supplementary Figure 1). Taken together, these data suggest that the partial therapeutic efficacy of CBD against GBM is due to a subset of tumor cells upregulating antioxidant response genes and undergoing an adaptive reprogramming toward a resistant, MES phenotype. PMT has been previously documented to occur in GBM following radiation ${ }^{5}$ or antiangiogenic 


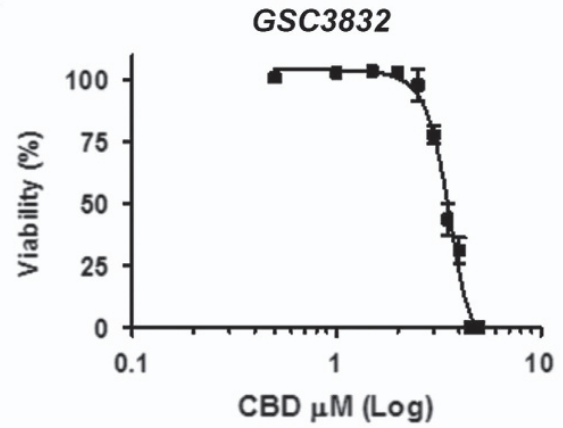

C

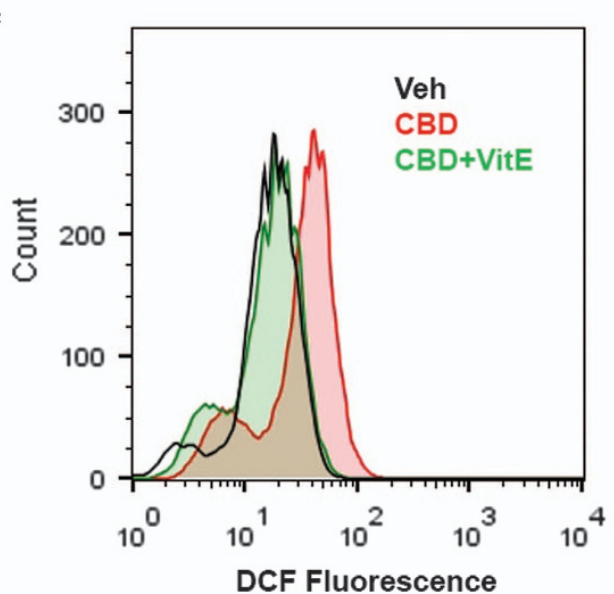

e

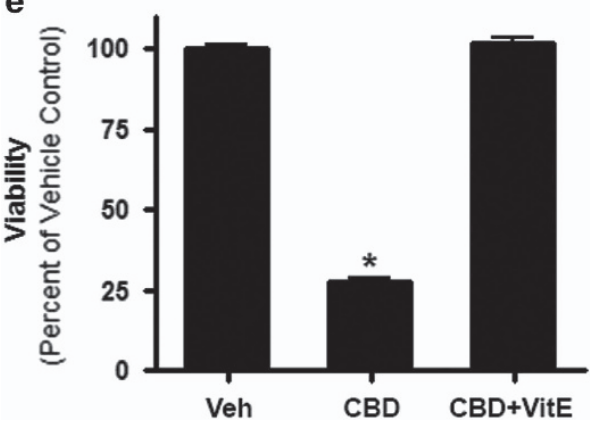

b

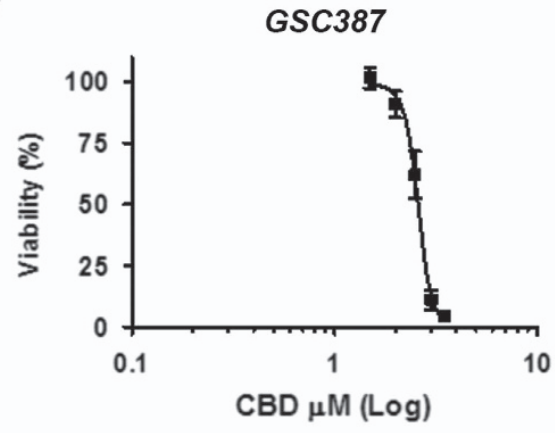

d

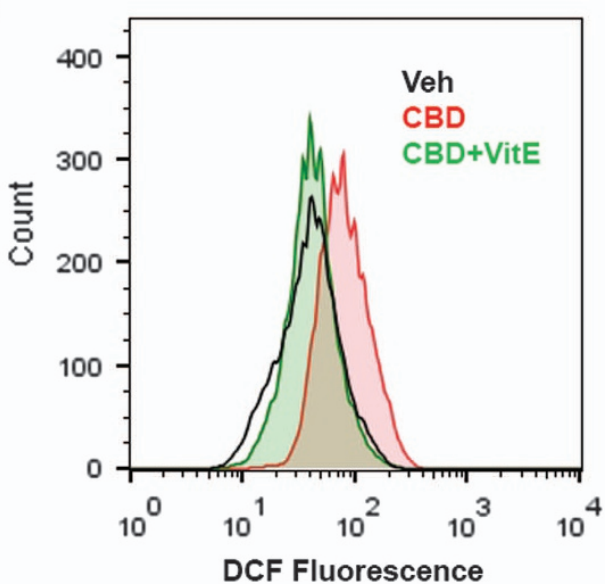

f

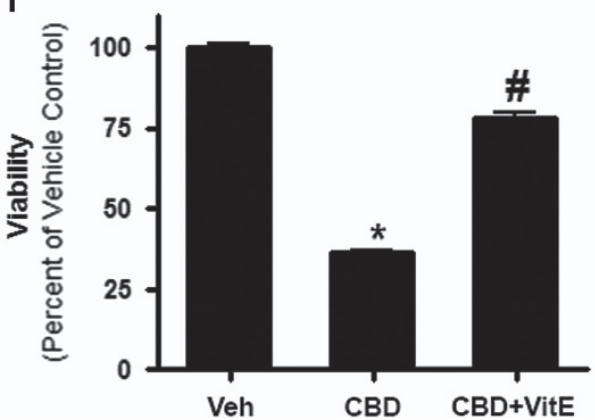

Figure $1 \mathrm{CBD}$ inhibits GSC viability and induces apoptosis through the production of ROS. (a and $\mathbf{b}) \mathrm{GSC}$ lines 3832 and 387 were treated with vehicle or CBD $(\mu \mathrm{M})$ for 2 days and cell viability was then evaluated. Viability (\%) was calculated as the Dojindo reagent product absorbance in the treated cells/control cellsx100. GSCs (c) 3832 and (d) 387 were treated with vehicle or $\operatorname{CBD}(2 \mu \mathrm{M})$ for 2 days and the production of ROS was measured using DCF and cell flow cytometry. (e and f) The contribution of ROS in CBDdependent $(2 \mu \mathrm{M})$ reductions in cell viability was evaluated using $40 \mu \mathrm{M}$ VitE as an antagonist. Data were compared using a one-way analysis of variance (ANOVA) with a corresponding Dunnett's post hoc test. ${ }^{*, \#}$ Statistically significant differences from control and CBD, respectively $(P<0.01)$

therapy. ${ }^{17}$ Given the significant upregulation of the antioxidant response gene products, we investigated whether the phenotypic shift toward a resistant, MES molecular profile was ROS-dependent. Cotreatment with ROS scavenger VitE partially reverted the CBD-induced MES shift measured in 3832 GSCs both at the transcript and protein levels (Figures $4 \mathrm{c}$ and $\mathrm{d}$ ). In agreement with these data, upregulation of the MES marker CD44 was measured specifically in GBM xenografts from CBD-treated mice, in vivo (Figure $4 \mathrm{e}$ ).

CBD-induced antioxidant response is mediated by NRF2 activation. Analysis of transcription factor activation, predicted from the analysis of microarray data using Altanalyze software indicated significant activation of the NFE2L2 (NRF2) transcriptional network (Figure 5a). To confirm the activation of NRF2 in another GSC line, we used 3832 cells treated with $\mathrm{CBD}$ or $\mathrm{CBD}+\mathrm{VitE}$. Western blot analysis $48 \mathrm{~h}$ following treatment demonstrates that the expression levels of the NRF2 targets SLC7A11 (xCT) and HMOX-1 were upregulated and these effects were reversed by VitE (Figure 5b). Next, we sought to investigate the molecular mechanism underlying NRF2-mediated activation of xCT and the overall antioxidant response program induced by CBD. In resting conditions, NRF2 is held inactive in the cytoplasm by KEAP1, being translocated to the nucleus following oxidative injury or stress. ${ }^{18}$ We used western blot analyses of 


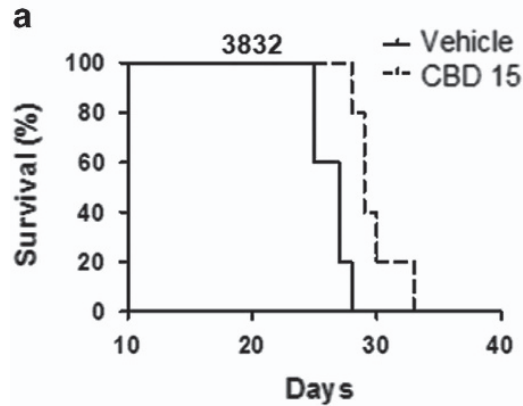

C

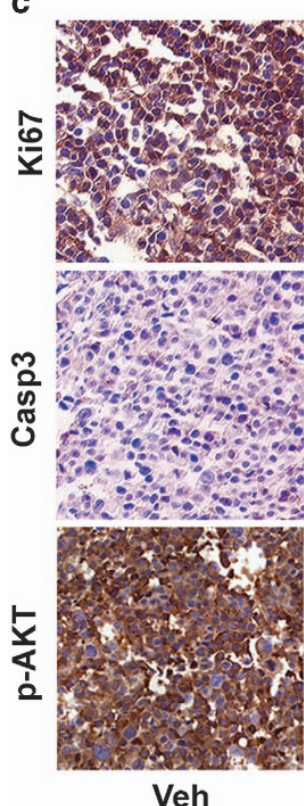

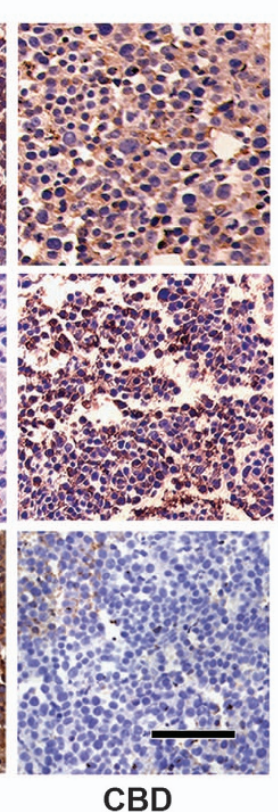

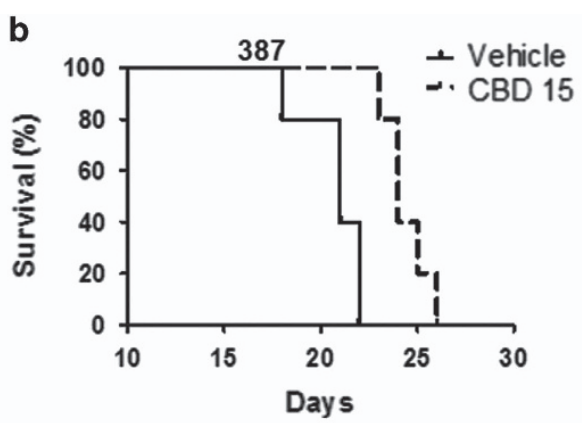

d

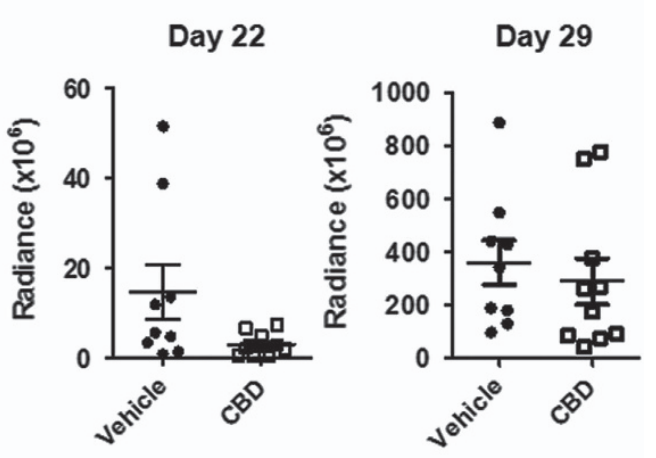

Figure 2 CBD prolongs survival mice bearing intracranial tumors derived from GSCs. Tumors were generated in female athymic nu/nu mice by the intracranial injection of $5 \times 10^{3}$ cells (a) 3832 or (b) 387 luciferase-labeled cells in $4 \mu$ l of media. Treatments were started at day 9 following BLI confirmation of tumor presence (using IVIS Lumina) and randomization of mice. Starting on day $9,15 \mathrm{mg} / \mathrm{kg}$ of CBD was administered intraperitoneally 5 days a week until completion of the experiment. Animal health was monitored daily and when mice showed signs of disease progression, they were humanely killed in accordance with institutional IACUC guidelines. Survival data was evaluated using Kaplan-Meier curves and a long-rank Mantel-Cox test. (c) Representative tumor sections represent inhibition of Ki67 (upper panel) staining, upregulation of cleaved caspase-3 (middle panel) and inhibition of p-AKT (lower panel) induced by CBD. Tumors were harvested 25 days after tumor induction. Bar $=200 \mu$ m. (d) BLI measurements demonstrate initial response to CBD (reduction of tumor size at day 22), followed by tumor resistance to treatment 1 week later (day 29)

subcellular fractions from CBD-treated GSC 3832, and determined that upon CBD treatment, the nuclear fraction of NRF2 was increased, an effect reversed by VitE (Figures $5 \mathrm{c}$ and $\mathrm{d}$ ). Promoter activation assays confirmed that CBD induced NRF2 activation in a concentration-dependent manner and this effect was reversed by VitE (Figure 5e). Taken together, these data indicate that CBD induces NRF2 activation, which in turns induces antioxidant response genes. Importantly, IHC analyses of xenograft tumor tissues from glioma bearing mice demonstrated in vivo upregulation of NRF2 and $\mathrm{xCT}$ in CBD treated as compared with vehicletreated tumors (Figure 5e), suggesting that this may be a key pathway underlying resistance to the CBD-based redox therapeutic.

Genetic targeting of SLC7A11 inhibits GSC self-renewal and cooperates with $C B D$ to inhibit cell survival. Given the robust upregulation of SLC7A11, we hypothesized that targeting its expression or function would revert some of the adaptive antioxidant response and thus render a more efficient therapeutic response in GSCs. We next used a pool of small interfering RNA (siRNA) sequences to target SLC7A11 in GSC 387, and measured the effects on cell viability and reduced glutathione (GSH) levels. We found that SLC7A11 knockdown inhibited GSH levels and these effects were reverted by pretreatment with the $\mathrm{N}$-acetyl cysteine antioxidant (Supplementary Figures $3 a$ and b). SLC7A11 knockdown cells showed enhanced sensitivity to CBD as compared with control siRNA-treated GSCs (Supplementary Figure $3 c$ ). To stably inhibit the expression levels of SLC7A11, we used a lentivirus-mediated knockdown, using two distinct short hairpin RNA (shRNA) sequences. Protein knockdown was significant (Supplementary Figure 3d) and the $x$ CT-knockdown GSCs were impaired in their ability to form tumorspheres (Supplementary Figures $3 e$ and f). Attempts to expand xCT-knockdown cells for in vivo studies 
a

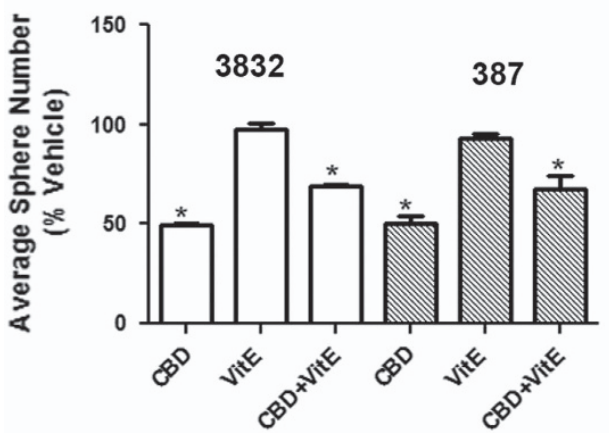

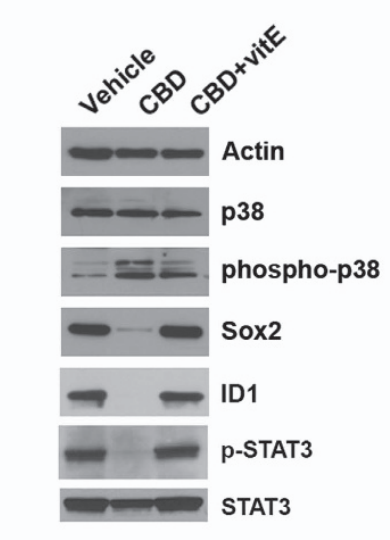

b
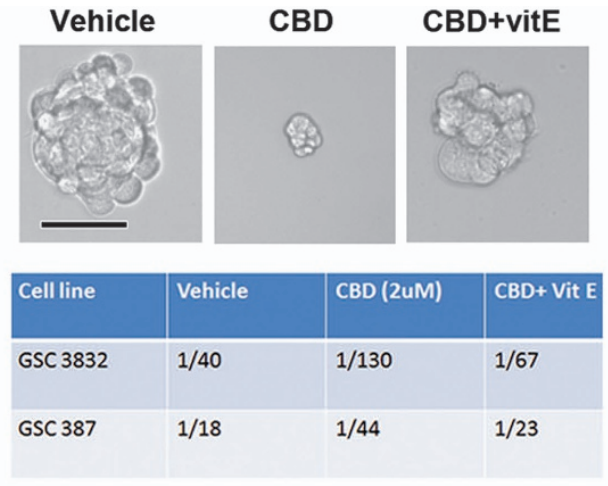

d

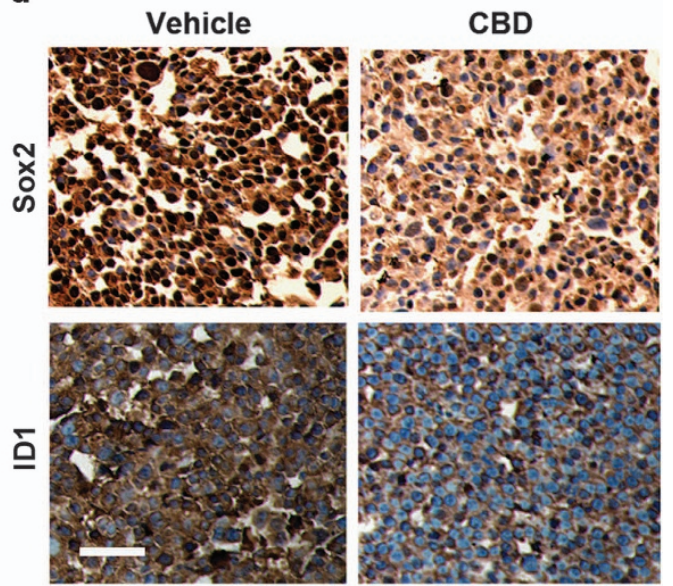

Figure 3 CBD inhibits GSC self-renewal. (a) GSC lines 3832 and 387 were subjected to sphere formation assays and sphere numbers were recorded 10 days later. *, \#P<0.01 for $2 \mu \mathrm{M} \mathrm{CBD}$ and $2 \mu \mathrm{M} \mathrm{CBD}+40 \mu \mathrm{M}$ VitE, respectively, compared with vehicle. The experiment was repeated two times. (b) Sphere frequency was measured using a limited dilution assay in both GSC lines treated with $\mathrm{CBD} \pm$ VitE. Upper panel shows representative photomicrographs in the indicated conditions. Lower panel table shows median stem cell frequency calculated using the ELDA software. (c) Western blot analyses in 3832 cells treated with $2 \mu \mathrm{M} \mathrm{CBD}$ or CBD+VitE for $48 \mathrm{~h}$, using the indicated antibodies. Activation of the p-p38 pathway concomitantly with the downregulation of the self-renewal master regulators, p-STAT3, Sox2 and Id1, are shown. (d) IHC analyses of xenograft glioma tissues from mice treated with vehicle or CBD (22 days). Staining with Sox2 and ID1 antibodies are shown. Bar $=100 \mu \mathrm{m}$

were not successful. As previously reported for cell lines, ${ }^{19}$ pharmacological inhibition of xCT inhibited reduced GSH levels in GSC 3832 (Supplementary Figure 4).

Pharmacological targeting of $\mathrm{xCT} /$ system Xc inhibits GSC survival, in an additive manner with CBD. Analysis of multiple PN and MES GBM samples demonstrated MES gliomas consistently expressed higher levels of $\mathrm{xCT}$ (Supplementary Figure $5 \mathrm{a}$ ). These results suggest that the upregulation of $x C T$ occurs in more aggressive GBM tumors. We next wished to pharmacologically inhibit $x C T$ using sulfasalazine (SAS), which has been previously shown to inhibit its function. ${ }^{19}$ SAS inhibited tumorsphere formation in GSCs by $\sim 40 \%$ (Supplementary Figure $5 b$ ). Concentrationresponse curves showed that SAS inhibited GSC 3832 and 387 cell viability with $I_{50}$ values of $564 \mu \mathrm{M}(546-582)$ and $548 \mu \mathrm{M}$ (522-575), respectively, demonstrating limited potency of the drug in culture (Supplementary Figures $5 \mathrm{c}$ and d). At these concentrations, SAS had limited solubility. We next measured the effect of combining CBD and SAS to target GSCs. Even under suboptimal conditions (due to SAS precipitation at high concentration), the combination of SAS enhanced the activity of CBD in an additive manner (Supplementary Figures $5 \mathrm{c}$ and e). Western blot analysis of SAS-treated GSC cultures demonstrated inhibition of proliferation markers (TOP2A) and upregulation of $\mathrm{xCT}$ (Supplementary Figure 5f). Administration of SAS in vivo was ineffective at targeting intracranial tumors derived from GSC 3832 cells because of its limited solubility and lack of potency. As shown in Supplementary Figure 5h, the levels of SLC7A11 were not upregulated in intracranial tumors derived from GSC 3832 cells during treatment with SAS, unlike the cultured GSCs, which have direct access to SAS. Given the limitations of administering SAS at effective concentrations in vivo to target GSCs, we turned our attention to a recently discovered novel class of system Xc inhibitors, Erastin (ERA), and its analog, piperazine erastine (PE). Both drugs showed efficacy in inhibiting system $X_{c}$ in several cancer cell lines and induced cell death via an iron-dependent mechanism, which is named as ferroptosis, in culture and in a subcutaneous xenograft cancer model. ${ }^{20-22}$ Although neither drug is able to cross the blood-brain barrier, their improved potency for targeting $\mathrm{xCT}$ made them important tools for 
a

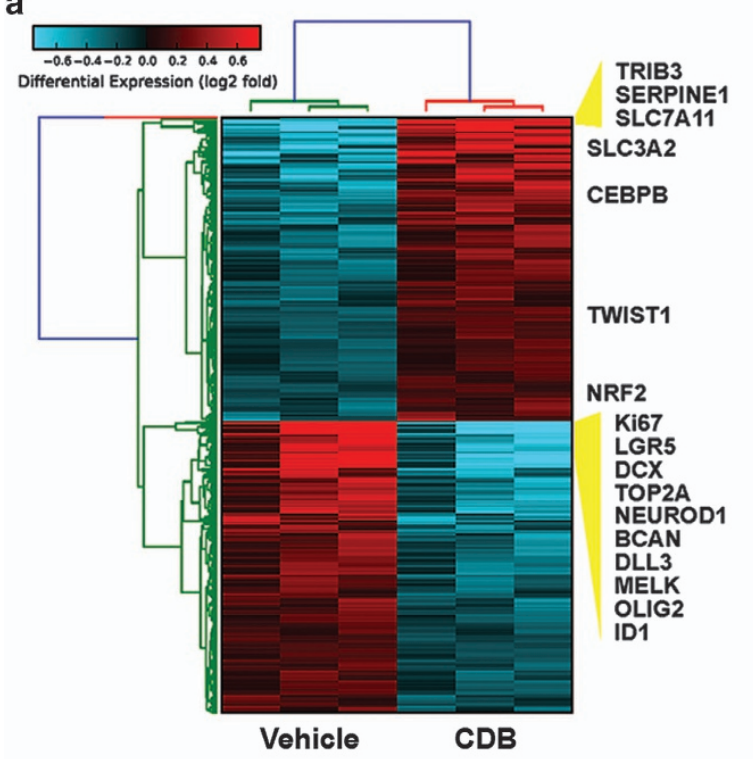

C

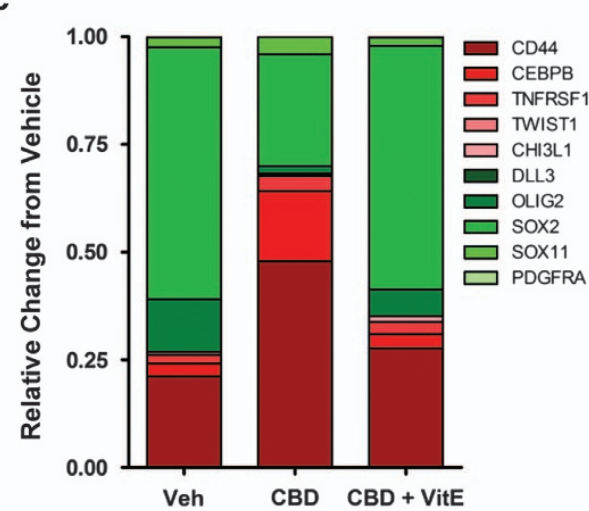

b

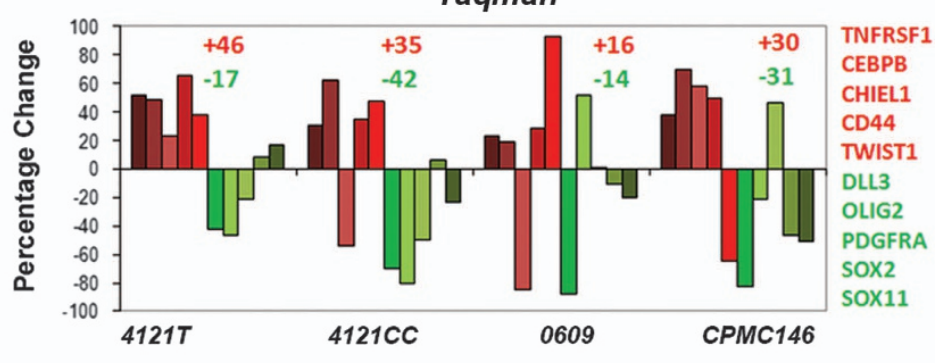

d

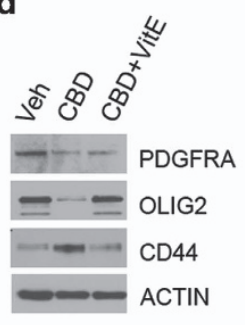

e

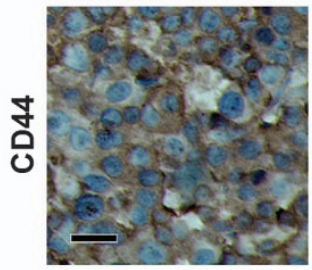

Veh

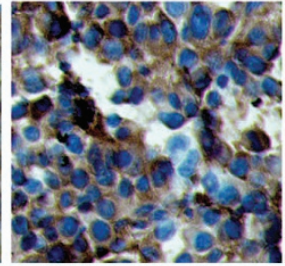

$C B D$

Figure 4 CBD treatment induces ROS-dependent PMT in GBM. (a) CBD treatment results in large-scale differential gene expression. Gene expression as log 2 fold changes, normalized between vehicle- and CBD- $(2 \mu \mathrm{M})$ treated groups for each cell line, are shown for 2107 protein coding and 243 noncoding RNAs (fold $>1.25$ and false discovery rates (FDR)-adjusted $t$-test, $P<0.1$ ). Predicted crucial regulatory factors are indicated on the right of the graph. Red depicts upregulation, while blue indicates downregulation. (b) Taqman validation of several PN (indicated in green) and MES (in red) markers in three additional GSC lines and an acutely dissociated patient tissue (CPMC146) confirm CBD-induced PMT. (c) Taqman analysis of PN and MES markers in 3832 cells treated with CBD or CBD+VitE. Results were normalized to Rab14 expression levels. (d) The 3832 GSCs treated with CBD \pm VitE were subjected to western blot analysis using the indicated antibodies to detect PN (Olig2) and MES (CD44) markers. (e) IHC analysis of GBM xenograft tissue sections from mice treated with vehicle or CBD (intraperitoneally $15 \mathrm{mg} / \mathrm{kg}, 5$ days per week). Tumor tissue was harvested at 29 days after tumor implantation. $\operatorname{Bar}=50 \mu \mathrm{m}$

further evaluating combination therapy in culture with our cannabinoid-based redox therapeutic, CBD.

Novel system Xc inhibitors ERA and PE inhibit GSC viability in a dose-dependent manner and act synergistically with CBD to inhibit GSC viability. ERA and PE inhibited GSC 387 viability with an $I_{50}$ value of $11.1 \mu \mathrm{M}$ (10.5-11.8) and $10 \mu \mathrm{M}(9.5-10.5)$, respectively (Figures 6a and c). In 387 cells, the inhibitory effects of ERA and PE were partially reverted by the iron chelators deferoxamine mesylate (DFO) and ferrostatin (Fer) (Figures $6 \mathrm{~b}$ and $\mathrm{d}$ ), demonstrating that these small molecules exert their antitumor effect along the same pathways as described for other cancers. ${ }^{20,21}$ PE upregulated PTGS2 (prostaglandin-endoperoxidase synthase 2) levels in GSC and this effect was reversible by VitE and DFO (Figure 6e). PTGS2 is a pharmacodynamic marker of system $X c$ inhibition. ${ }^{20-22}$

Importantly, CBD in combination with ERA led to a synergistic increase in the inhibition of GSC viability (Figures $7 a$ and $b$ ). The effect on viability with the combination of CBD+ERA correlated with an enhanced production of ROS (Figures $7 \mathrm{c}$ and d). Furthermore, we performed two functional assays, which measure pathognomonic features of glioma, that is, invasion and self-renewal. Matrigel invasion assays demonstrate that $\mathrm{CBD}$ and ERA synergistically inhibited tumor cell invasion, as shown in Figures $7 e$ and $\mathrm{f}$. Self-renewal was assessed using a limited dilution assay, which demonstrated that treatment with $\mathrm{CBD}+\mathrm{ERA}$ significantly downregulated GBM stem cell frequency in two GSC cell lines (Figures $7 \mathrm{~g}$ and $\mathrm{h}$ ). The combination treatment was more efficient than either drug used alone (Figures $7 \mathrm{~g}$ and $\mathrm{h}$ ). We have also tested the 


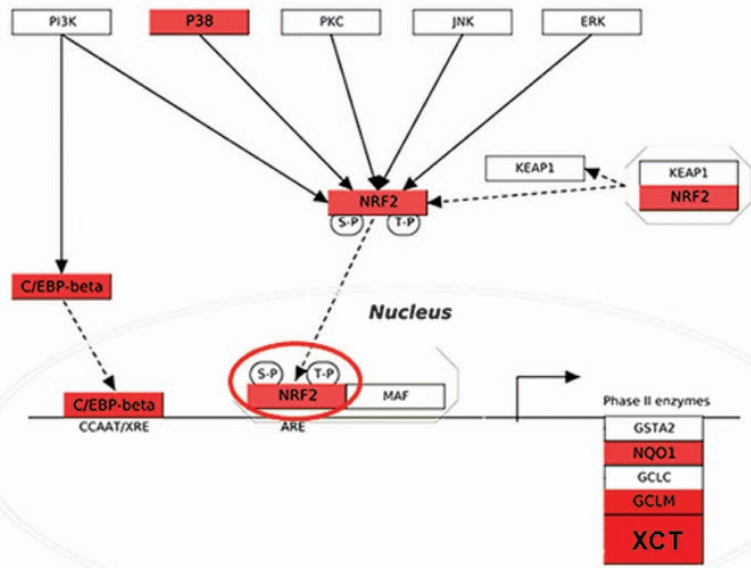

C

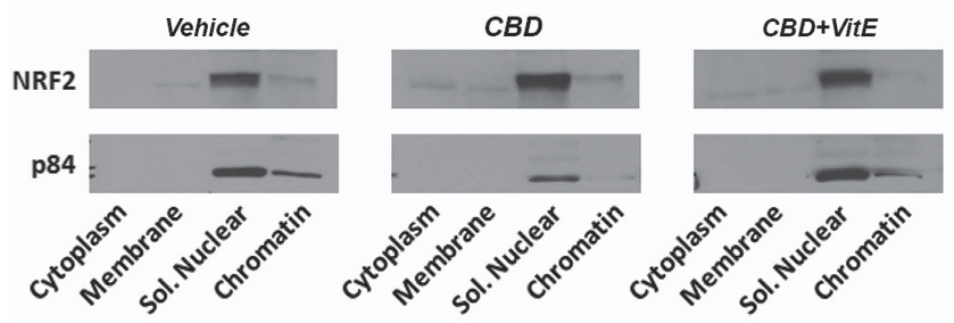

e

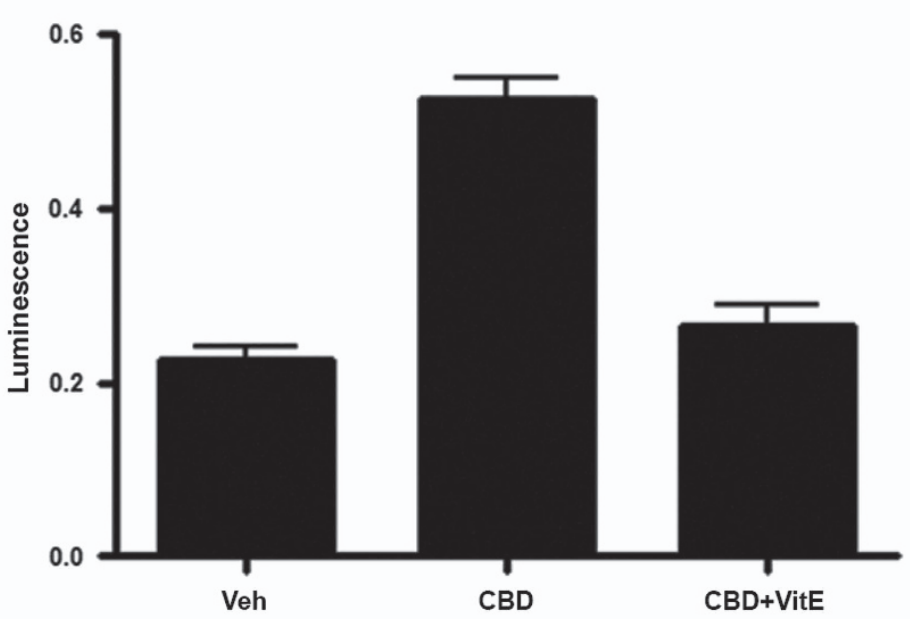

b
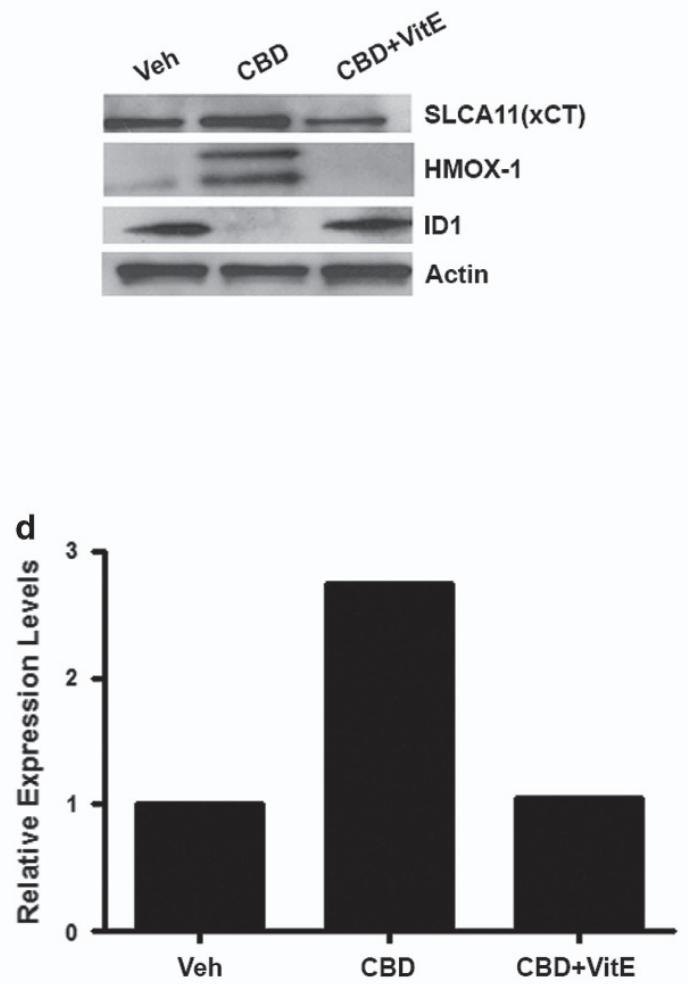

f

Veh

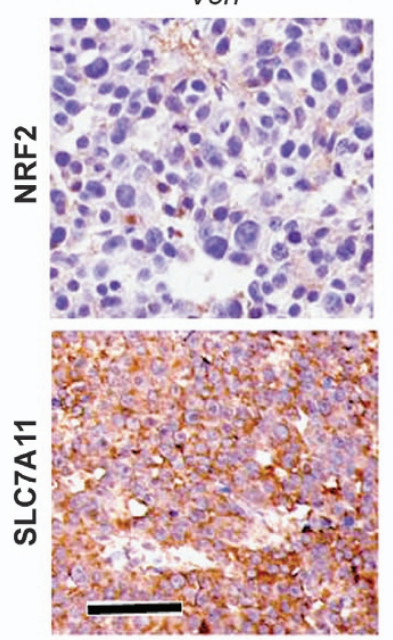

$C B D$

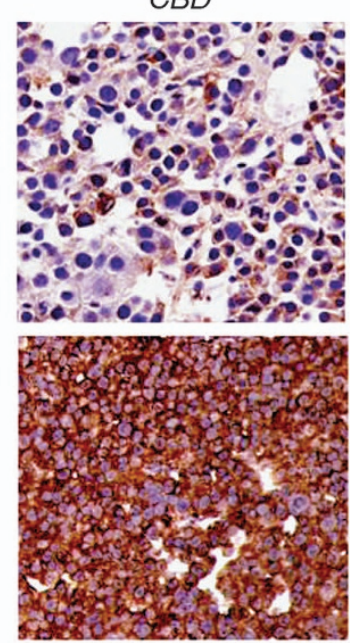

Figure $5 \mathrm{CBD}$ induces nuclear translocation and activation of NRF2 and its downstream targets. (a) Visualization of CBD-associated gene expression changes along the NRF2 transcriptional network. Molecules shown in red were significantly upregulated by CBD $(>2 \times)$. (b) Western blot analysis confirms ROS-dependent upregulation of NRF2 target proteins HMOX-1 and $\mathrm{xCT}$, following $48 \mathrm{~h}$ treatment with $\mathrm{CBD} \pm 40 \mu \mathrm{M}$ VitE. (c) Subcellular fractionation using GSC 3832 cell line treated with $\mathrm{CBD} \pm$ VitE was used to generate protein lysates for western blot with the indicated antibodies. (d) Quantification of NRF2 levels using densitometry shows that relative to $\mathrm{p} 84$ (control), nuclear levels of NRF2 are enhanced by CBD treatment. (e) GSCs were transfected using the Qiagen Cignal Antioxidant Luciferase Reporter Kit (Qiagen Inc, Valencia, CA, USA; no. CCS-5020L) and Lipofectamine 2000 (Life Technologies) in OptiMEM media and 1\% NEAA. At $24 \mathrm{~h}$ after transfection, the cells were treated with vehicle, $2.0 \mu \mathrm{M}$ CBD, $2.0 \mu \mathrm{M}$ CBD+40 $\mu \mathrm{M}$ VitE or DL-sulforapahane $(40 \mu \mathrm{M})$ the manufacturer's recommended positive control for $48 \mathrm{~h}$. NRF2 activity was measured using the Promega Dual-GLO Luciferase Assay system (Promega Corporation, Sunnyvale, CA, USA). First, NRF2-dependent luminescence was measured and then constitutively expressed luminescence was measured to normalize for cell number. The plotted luminescence units are a ratio of the two readings. Data were compared using a one-way analysis of variance (ANOVA) with a corresponding Dunnett's post hoc test. ${ }^{*, \#}$ Statistically significant differences from control and CBD at $P<0.05$, respectively. (f) IHC detection of NRF2 and SLC7A11 (xCT) in GBM xenograft tissues from mice treated with vehicle or CBD for 22 days. Bar $=200 \mu \mathrm{m}$ 


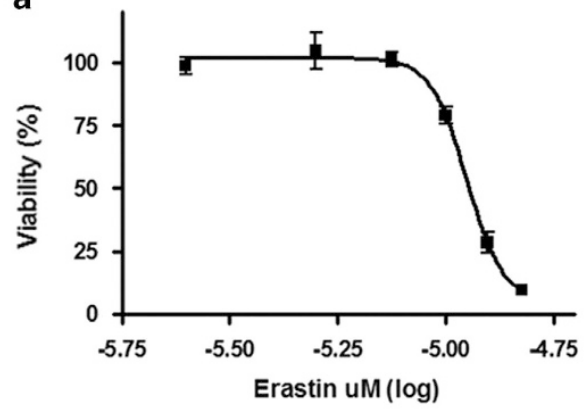

b

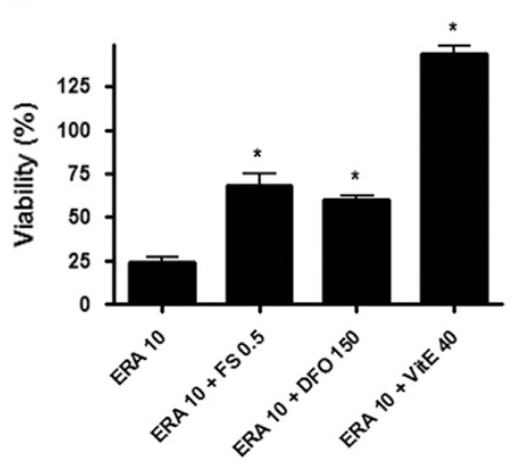

c

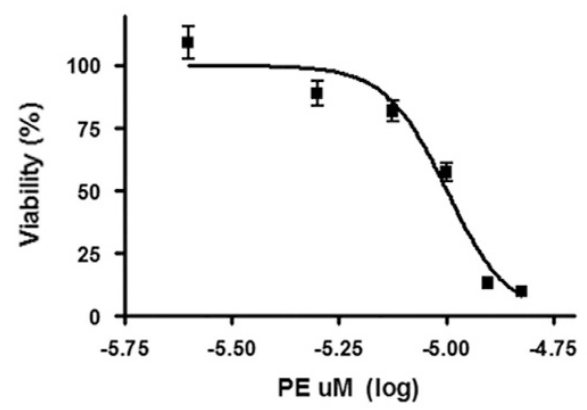

d

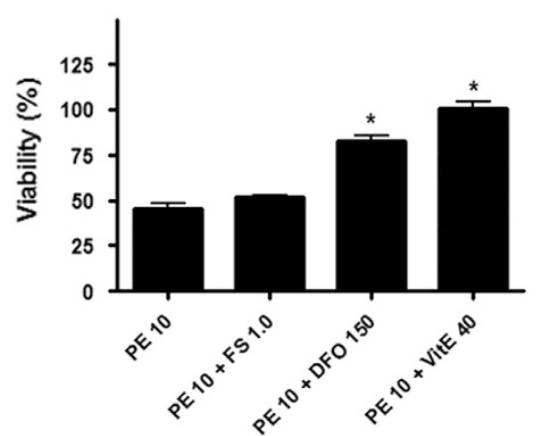

e

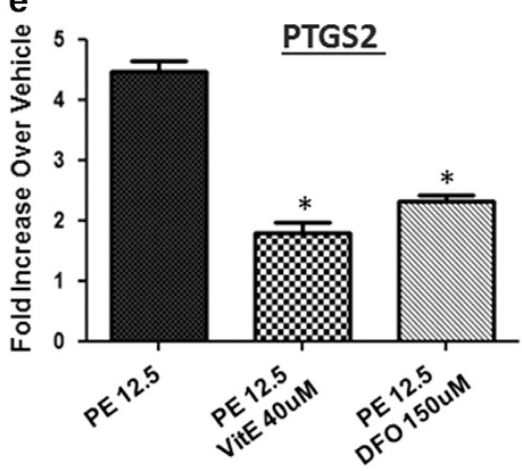

Figure 6 ERA and PE inhibit GSC viability in an iron-dependent manner. (a and c) GSCs 387 were treated with $\mu \mathrm{M}$ concentrations of ERA or PE for 5 days and the percentage of cell viability was calculated as the Dojindo reagent product absorbance in the treated cells/control cells $\times 100$. (b) GSC 387 were treated with indicated $\mu \mathrm{M}$ concentrations of ERA $\pm \mathrm{DFO}$, Fer or VitE, as indicated. (d) GSC 387 was treated with shown $\mu \mathrm{M}$ concentrations of PE $\pm \mathrm{DFO}$, Fer or VitE. (e) Taqman determination of PTGS2 levels after GSC 387 treatment with $P E \pm V i t E$ and DFO. ${ }^{*} P<0.02$ ANOVA

combination of PE and CBD on GSC 387 viability and our results demonstrate that the two drugs act synergistically to inhibit tumor cell viability (Supplementary Figure 6). Supplementary Table 1 summarizes combination index $(\mathrm{Cl})$ values for $\mathrm{CBD}$ and various system $\mathrm{Xc}$ inhibitors in inhibiting GSC survival, where a $\mathrm{Cl}$ value of $<1,1$ and $>1$ indicates synergistic, additive and antagonistic effects, respectively.

\section{Discussion}

To study the response of GSC to a redox therapeutic that stimulates ROS, we used the non-toxic, non-psychoactive cannabinoid, CBD. CBD has been previously shown to inhibit GBM progression in subcutaneous and intracranial models representing bulk tumor mass. ${ }^{14,23,24}$ CBD has also been shown to inhibit the progression of other cancers in vivo. ${ }^{25-28}$ The most unifying mode of action in culture for these effects has been the selective production of ROS in tumor cells as opposed to non-transformed cells. ${ }^{11}$ However, whether the ability of CBD to inhibit cancer aggressive through the production of ROS in culture is linked to the antitumor activity of $\mathrm{CBD}$ in vivo has not been established.

In this investigation, CBD-dependent inhibition of GSC viability in culture was mediated by an increase in the production of ROS, which could be reverted by the antioxidant VitE.

CBD-induced therapeutic benefit can be attributed, in part, to the inhibition of GSC self-renewal and stemness as shown by functional assays and biomarker analyses in xenograft tissues. Our results confirm the recent report that p-p38 activation underlies ROS-mediated reprogramming of GSCs ${ }^{29}$ and furthermore show for the first time that stem cell key regulators such as Id1, Sox2 and p-STAT3 are inhibited by CBD in an ROS-dependent manner. These findings are relevant in the context of using CBD as adjuvant therapy for GBM, to complement the standard of care (e.g., Temodar, radiation), which is not efficacious against GSCs. ${ }^{30}$

CBD was able to inhibit GBM progression in vivo and prolonged survival. IHC analysis of tumor tissue demonstrated CBD-dependent inhibition of cancer cell proliferation and induction of apoptosis, as assessed by decreased Ki67 and increased cleaved caspase-3 staining, respectively. Overall, however, the inhibitory effects on GSC progression in vivo were not as robust as when using standard glioma lines such as $U 87^{23}$ and $U 251^{13}$ cells. This provided us with an excellent opportunity to understand GSC adaptive changes to a redox therapeutic.

Using DNA microarrays, we interrogated changes occurring in GSCs following CBD treatment. Data analysis demonstrated that CBD significantly inhibited the expression of stem cell regulators and GBM PN markers, with a concomitant upregulation of antioxidant response gene products and MES markers. Using western blot assays, we discovered that induction of the antioxidant response and the MES phenotype were both reversible by antioxidant pretreatment, suggesting a 
a

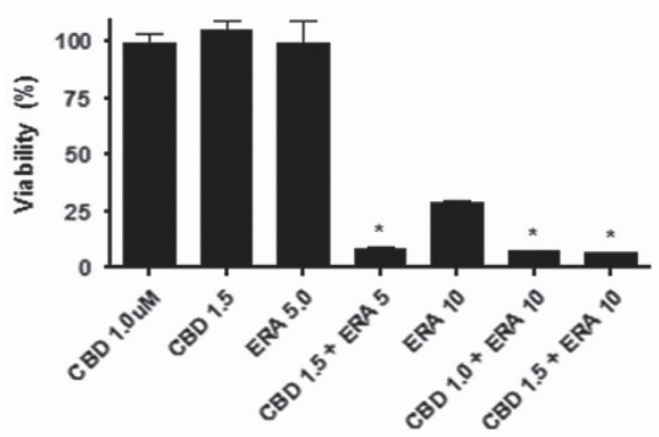

d

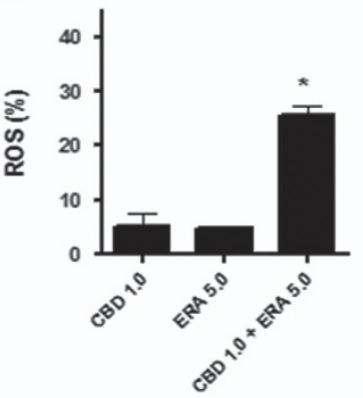

b

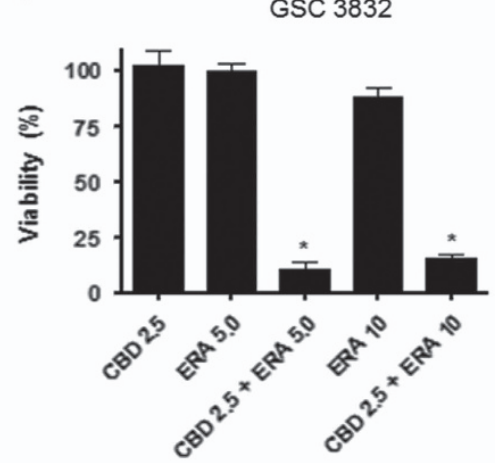

e

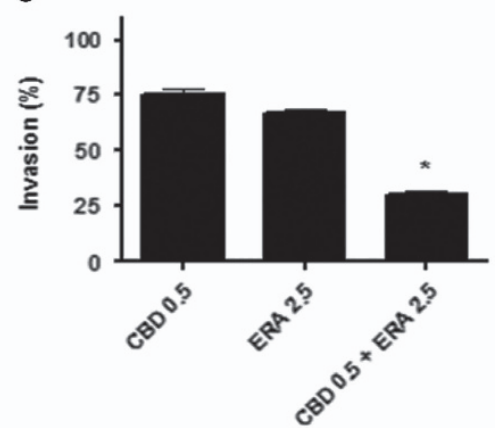

C

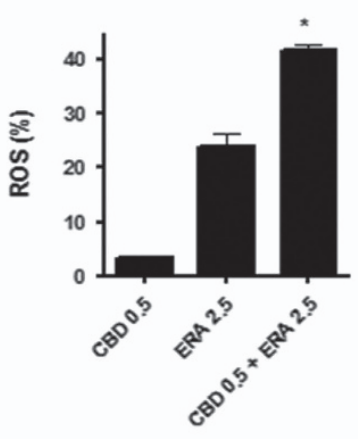

f

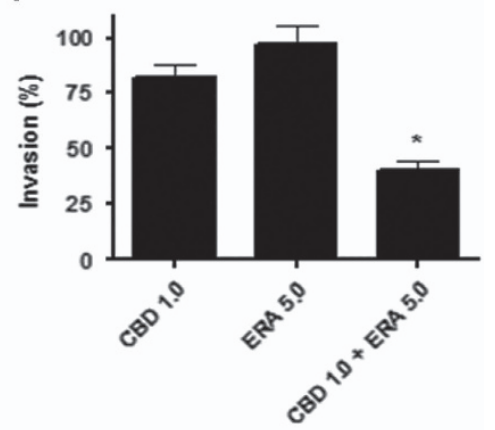

g

\begin{tabular}{|l|l|l|}
\hline Treatment & GSC\#3832 & GSC\#387 \\
\hline Vehicle & $1 / 38$ & $1 / 13$ \\
\hline CBD & $1 / 246$ & $1 / 200$ \\
\hline ERASTIN & $1 / 183$ & $1 / 130$ \\
\hline CBD+ERASTIN & $1 / 375$ & $1 / 332$ \\
\hline
\end{tabular}

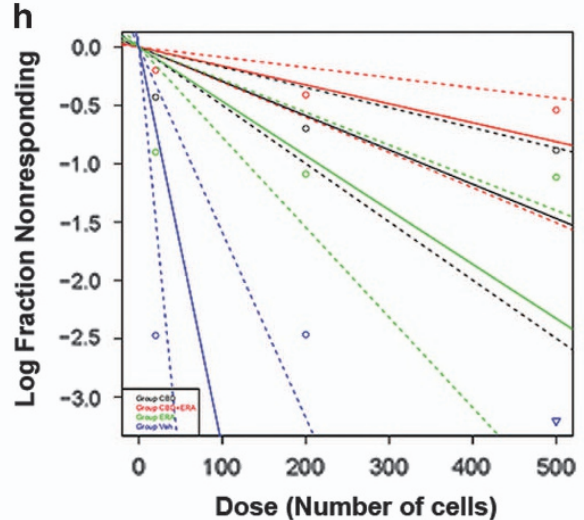

Figure 7 CBD and ERA synergistically inhibit GSC viability, invasion and self-renewal. (a and $\mathbf{b})$ GSC lines 387 and 3832 were treated with CBD, ERA or CBD+ERA ( $\mu$ M). Viability (\%) was calculated as the Dojindo reagent product absorbance in the treated cells/control cells $\times 100$. These data were used to calculate a $\mathrm{Cl}$ value $(0.53$ and 0.49 for GSC 387, and 0.89 and 0.85 for GSC 3832) for the two combined concentrations in each group, where a $\mathrm{Cl}$ value of $<1,1$ and $>1$ indicates synergistic, additive and antagonistic effects, respectively (see also Supplementary Table 1). (c and d) ROS measurements show the combination of CBD+ERA ( $\mu$ M) enhanced ROS production in the 3832 and 387 GSC lines. ROS was measured using 2',7'dichloro-dihydrofluorescein and cell flow cytometry. The \% increase in ROS was calculated as the FL2 emission shift in treated cells/vehicle cells $\times 100$. (e and f) Invasion assays were performed using GSC 387, GSC 3832 in the presence of vehicle, CBD, ERA and combination of CBD+ERA ( $\mu \mathrm{M}$ ). Invasion (\%) was calculated as the number of cells invading in the treated cells/control cells $\times 100$. Data were compared using a one-way analysis of variance (ANOVA) with a corresponding Dunnett's post hoc test. *Statistically significant differences from control at $P<0.05$. (g and $\mathbf{h})$ Limited dilution assays were performed using 3832 and 387 GSCs cultured in three decreasing cell densities. Inhibitors were added at the time of initial culturing (CBD $=1.5 \mu \mathrm{M}, \mathrm{ERA}=5 \mu \mathrm{M})$. The number of wells with spheres was recorded 10 days following initial culturing and data were analyzed using ELDA software. The plot represents analysis for 387 cells; the chart includes estimated (median) stem cell frequency for both GSC lines in the presence of CBD, ERA and CBD+ERA $(\mu \mathrm{M})$ as indicated. Overall test for stem cell frequency between any of the groups shown in $(\mathbf{g}$ and $\mathbf{h})$ had a $P$-value of $2.2 e-5$

dependence on ROS. More specifically, we measured the activation of a critical antioxidant response element (ARE) activator, NRF2. Upon sensing of increase ROS levels, NRF2 is released from its complex with KEAP1, and it translocates to the nucleus, where it binds ARE found in the promoters of ROSdetoxifying enzymes, including NQO1, GST, HMOX-1 and $\mathrm{xCT}^{18,31}$ Importantly, augmented expression of both NRF2 and
$\mathrm{xCT}$ was observed in vivo in CBD-treated tumor tissue, as compared with vehicle. This demonstrates for the first time that CBD targeting of GSC results in the activation of pathways responsible for counteracting oxidative stress in vivo.

Cysteine metabolism, regulated by $x C T$, is a critical determinant of GBM growth and invasion. Interestingly, recent studies have identified $\mathrm{xCT}$ as a key regulator of cancer cell 


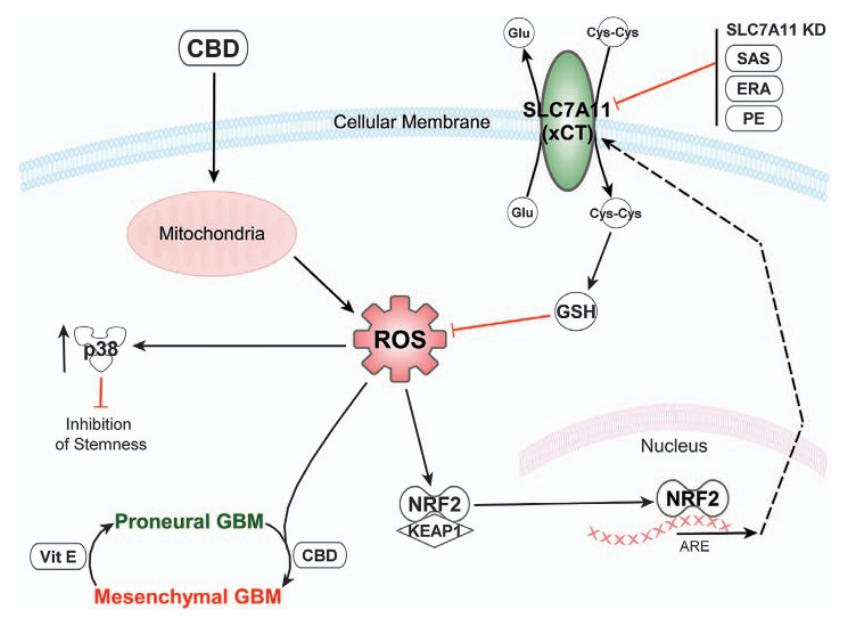

Figure 8 Diagram summarizes adaptive signaling pathways activated by CBD via ROS upregulation (PMT and antioxidant response, both reversible) in GSCs. Cotargeting using $\mathrm{CBD}$ and system $\mathrm{XC}(\mathrm{XCT})$ inhibitors synergistically inhibit GSC viability. The diagram was produced using the Ingenuity Pathway Analysis software

metabolic reprogramming and therapeutic target in gastric cancer and in triple-negative breast carcinomas. ${ }^{32,33}$ In this study, we explored the utility of small-molecule inhibitors of $\mathrm{xCT}$ as antiglioma agents. SAS, previously used in other published reports, ${ }^{19}$ exhibited limited antitumor activity as monotherapy, and acted in an additive manner with CBD. Because of its low potency and limited solubility, combination experiments in xenograft models could not be performed. We next tested a new generation of system Xc inhibitors, ERA and PE. ERA has been identified through a screen for small molecules efficacious against Ras-driven cancers and modulates intracellular levels of GSH and ROS. ${ }^{34}$ This is the first study to report the activity of ERA against primary GSCs. In other cancers, cell killing by ERA occurs as a novel irondependent, cellular death mechanism, which is named as ferroptosis. ${ }^{21}$ Our studies using specific iron chelators demonstrate that in GBM cells, ERA works akin to inhibition of other cancers. As ERA has low solubility in organic solvents, $\mathrm{PE}$ has been recently synthesized and characterized as an alternative to ERA for targeting tumor cells. ${ }^{21}$ We showed that $P E$ is efficacious against GBM cells and this effect can be partially reverted by iron chelators. Importantly, both ERA and $P E$ act synergistically with CBD to inhibit viability of GSC. However, unlike the case of flank tumors, PE does not cross the blood-brain barrier and as such was not suitable for in vivo testing in our orthotopic GBM model. Novel PE derivatives with better blood barrier penetrance and alternate delivery approaches are currently under investigation.

Our data presented herein demonstrates for the first time that administration of CBD can inhibit intracranial growth of primary GSC-derived tumors in vivo and this effect is mediated by an increase in ROS levels. Our results support the notion that CB-based therapeutics in combination with other non-toxic small-molecule inhibitors of antioxidant response genes can synergistically inhibit GBM progression and should be considered for the development of novel therapeutics. The diagram in Figure 8 summarizes adaptive signaling pathways modulated by $C B D$ via ROS up-regulation in glioma stem cells.
Materials and Methods

Cell lines and reagents. The U251 cell line was obtained from ATCC (Manassas, VA, USA). GSC lines 387 and 3832 were generously provided by $\mathrm{Dr}$ Jeremy Rich, while all other GSC lines used were generated in-house from tissue samples obtained during surgical resection of patients diagnosed with GBM, using an IRB-approved protocol. Tumors were then subjected to enzymatic digest, mechanically dissociated and cultured as neurospheres as previously described by our group. Tumor lines were maintained as subcutaneous flank xenografts in athymic Nu/Nu mice and processed as stated above. All GSC lines were cultured in growth media made up of Neurobasal Media (Lifetech, Chicago, IL, USA) supplemented with N2 (Lifetech), GlutMAX (Lifetech), basic fibroblast growth factor and epidermal growth factor, both at $25 \mu \mathrm{g} / \mathrm{ml}$ (Promega Corp., Madison, WI, USA), unless stated otherwise. CBD was obtained from the National Institutes of Health through the National Institute of Drug Abuse. Ethanol served as the vehicle control in all culture studies. Tocopherol (TOC; Sigma-Aldrich, St. Louis, MO, USA), SAS (Sigma-Aldrich), Fer (Sigma-Aldrich), DFO (Abcam Ltd, Boston, MA, USA) and ERA (Selleck Chemicals, Houston, TX, USA) were all obtained commercially from indicated vendors. PE was generously donated by $\mathrm{Dr}$. Brent Stockwell (Columbia University, New York, NY, USA).

Cellular ROS detection using flow cytometry. ROS measurements were made by plating GBM cells at $0.175 \times 10^{6}$ cells per well in 6 -well plates precoated with GelTrex Reduced Growth Factor Basement Membrane Matrix (Life Technologies, Grand Island, NY, USA) for $3 \mathrm{~h}$ at $0.04-0.06 \mathrm{mg} / \mathrm{ml}$ (dependent on the manufacturer's stated concentration range). Cells were allowed to recover overnight. At $24 \mathrm{~h}$, media were aspirated and replaced with fresh growth media lacking GlutMAX (Lifetech) and supplemented with indicated drug concentrations and the addition of $2^{\prime}-7^{\prime}$ dichlorofluorescein diacetate (DCF; Sigma-Aldrich) at $10 \mu \mathrm{M}$. Cultures were incubated with treatment media for $12 \mathrm{~h}$, harvested with TrypLE Express (Lifetech), resuspended in $300 \mu \mathrm{l}$ PBS and ROS was assessed by cell flow cytometry. Representative histogram plots were made using FlowJo software (Tree Star Inc., Ashland, OR, USA).

Expression profiling using Human Gene 1.0 ST Affymetrix Arrays. Affymetrix Human Gene 1.0 ST arrays were processed according to the Affymetrix Expression Analysis Whole Transcript (WT) Sense Target Labeling Protocol (Affymetrix, Inc.). Briefly, total RNA (300 ng) was converted to doublestrand cDNA. CRNA was obtained by an in vitro transcription reaction and used as the template for generating a new first-strand cDNA. The cDNA was fragmented, end-labeled with biotin and hybridized to the array for $16 \mathrm{~h}$ at $45^{\circ} \mathrm{C}$ using the GeneChip Hybridization Oven 640 (Affymetrix, Inc.). Washing and staining with streptavidin-phycoerythrin was performed using the GeneChip Fluidics Station 450 and the images acquired using the Affymetrix Scanner $30007 G$ Plus (Affymetrix, Inc.). The data were normalized using quantile normalization with the RMA algorithm for gene-level intensities and the ratio determined for each gene using Partek Genomics Suite (Partek Inc., St. Louis, MO, USA). Total RNA was processed at the microarray facility from the center for applied genomics at Public Health Research Institute in New Jersey, using the Affymetrix Gene 1.0 ST Array.

Human affymetrix data analysis. Mean values of selected human and HCMV transcripts in the IE1KD versus Control are displayed using the R program heatmap.2 from the package 'gplots'. The package is available from the $\mathrm{R}$ repository CRAN, and is maintained by Gregory R. Warnes. Raw data is available at http://www.ncbi.nlm.nih.gov/geo/query/acc.cgi?acc=GSE57978.

Additional analyses were performed using AltAnalyze software developed by Dr Nathan Salomonis and Ingenuity analysis software (Ingenuity IPA, Redwood City, CA, USA).

Limited dilution assays and sphere formation. CD133+ cells from primary GSC lines maintained on the flank of nude mice were plated in 96-well plates at 10, 100 and 1000 cells per well (12 wells/condition) in complete growth media. Cells were retrieved after sorting using MACS directly in Neurobasal media +growth factors and inhibitors were added directly to cells at the time of initial culturing. Tumorspheres were evaluated 10 days following initial culturing, using an inverted microscope fitted with a camera. Wells were scored positive when at least one sphere was present. The estimated stem cell frequency was estimated using the ELDA. For percent sphere formation, 100 GSCs were plated per well of a 24 well plate and sphere formation (at 7 days) was calculated using the following formula: percent sphere formation $(\%)=$ number of spheres/number of cells plated $\times 100$. Each condition was run in quadruplicate and the experiment was repeated two times. 
Orthotopic mouse model of GBM. Tumors were generated in female athymic nu/nu mice by the intracranial injection of luciferase-labeled GSC 3832 or 387 in $4 \mu \mathrm{l}$ of RPMl. Treatments were started at day 9 (arrow) following bioluminescence imaging (BLI) confirmation of tumor presence (using IVIS Lumina instrument, Perkin Elmer, Santa Clara, CA, USA) and randomization of mice. Starting on day 9, CBD was administered intraperitoneally 5 days a week until completion of the experiment. Animal health was monitored daily, and when mice showed signs of disease progression, they were humanely killed in accordance with institutional IACUC guidelines.

Data analysis and statistical procedures. All data shown represents two independent experiments with $\geq 3$ replicates. The $\mathrm{IC}_{50}$ values with corresponding $95 \%$ confidence limits were compared by the analysis of logged data (GraphPad Prism; Graphpad Software Inc., La Jolla, CA, USA). Significant differences were also determined using a one-way ANOVA or the unpaired Student's $t$-test, where suitable. Survival data was evaluated using Kaplan-Meir curves and a log-rank Mantel-Cox test. $P$-values $<0.05$ defined statistical significance. Statistical significance was assessed using GraphPad Prism (Graphpad Software Inc.).

\section{Conflict of Interest}

The authors declare no conflict of interest.

Acknowledgements. We are grateful to Dr. Charles Cobbs (The Ivy Center for Brain Tumor Research, Swedish Hospital, Seattle, WA, USA) for providing glioblastoma tissues and to Dr. Jeremy Rich (Cleveland Clinic, Clevelan, OH, USA) for the GSC lines 387 and 3832. We thank Dr. Brent Stockwell (Columbia University) for providing PE. This work was supported by NIH Grants R21NS067395 (LS), R01NS070289 (LS, CSC), and additional funding from the Flaming foundation, the $\mathrm{ABC} 2$ foundation and the CPMC Research Institute.

1. Phillips HS, Kharbanda S, Chen R, Forrest WF, Soriano RH, Wu TD et al. Molecula subclasses of high-grade glioma predict prognosis, delineate a pattern of disease progression, and resemble stages in neurogenesis. Cancer Cell 2006; 9: 157-173.

2. TCGA C. Comprehensive genomic characterization defines human glioblastoma genes and core pathways. Nature 2008; 455: 1061-1068.

3. Verhaak RG, Hoadley KA, Purdom E, Wang V, Qi Y, Wilkerson MD et al. Integrated genomic analysis identifies clinically relevant subtypes of glioblastoma characterized by abnormalities in PDGFRA, IDH1, EGFR, and NF1. Cancer Cell 2010; 17: 98-110.

4. Verhaak RG, Tamayo $P$, Yang JY, Hubbard D, Zhang H, Creighton CJ et al. Prognostically relevant gene signatures of high-grade serous ovarian carcinoma. J Clin Invest 2012; 123 517-525

5. Bhat KP, Balasubramaniyan V, Vaillant B, Ezhilarasan R, Hummelink K, Hollingsworth F et al. Mesenchymal differentiation mediated by NF-kappaB promotes radiation resistance in glioblastoma. Cancer Cell 2013; 24: 331-346.

6. Mao P, Joshi K, Li J, Kim SH, Li P, Santana-Santos L et al. Mesenchymal glioma stem cells are maintained by activated glycolytic metabolism involving aldehyde dehydrogenase $1 \mathrm{~A} 3$. Proc Natl Acad Sci USA 2013; 110: 8644-8649.

7. Bao S, Wu Q, McLendon RE, Hao Y, Shi Q, Hjelmeland AB et al. Glioma stem cells promote radioresistance by preferential activation of the DNA damage response. Nature 2006; 444 756-760.

8. Benhar M, Engelberg D, Levitzki A. ROS, stress-activated kinases and stress signaling in cancer. EMBO Rep 2002; 3: 420-425.

9. Wondrak GT. Redox-directed cancer therapeutics: molecular mechanisms and opportunities. Antioxid Redox Signal 2009; 11: 3013-3069.

10. Laurent A, Nicco C, Chereau C, Goulvestre C, Alexandre J, Alves A et al. Controlling tumor growth by modulating endogenous production of reactive oxygen species. Cancer Res 2005 65: 948-956.

11. Massi $\mathrm{P}$, Solinas M, Cinquina V, Parolaro D. Cannabidiol as potential anticancer drug Br J Clin Pharmacol 2012; 75: 303-312.

12. Velasco G, Sanchez C, Guzman M. Towards the use of cannabinoids as antitumour agents Nat Rev Cancer 2012; 12: 436-444.

13. Akhavan A, Griffith OL, Soroceanu L, Leonoudakis D, Luciani-Torres MG, Daemen A et al. Loss of cell-surface laminin anchoring promotes tumor growth and is associated with poor clinical outcomes. Cancer Res 2012; 72: 2578-2588.
14. Soroceanu L, Murase R, Limbad C, Singer E, Allison J, Adrados I et al. Id-1 is a key transcriptional regulator of glioblastoma aggressiveness and a novel therapeutic target. Cancer Res 2013; 73: 1559-1569.

15. Hu Y, Smyth GK. ELDA: extreme limiting dilution analysis for comparing depleted and enriched populations in stem cell and other assays. J Immunol Methods 2009; 347: 70-78.

16. Shi X, Zhang Y, Zheng J, Pan J. Reactive oxygen species in cancer stem cells. Antioxid Redox Signal 2012; 16: 1215-1228.

17. Lu KV, Chang JP, Parachoniak CA, Pandika MM, Aghi MK, Meyronet D et al. VEGF inhibits tumor cell invasion and mesenchymal transition through a MET/VEGFR2 complex. Cancer Cell 2012; 22: 21-35.

18. Magesh S, Chen Y, Hu L. Small molecule modulators of Keap1-Nrf2-ARE pathway as potential preventive and therapeutic agents. Med Res Rev 2012; 32: 687-726.

19. Lyons SA, Chung WJ, Weaver AK, Ogunrinu T, Sontheimer H. Autocrine glutamate signaling promotes glioma cell invasion. Cancer Res 2007; 67: 9463-9471.

20. Dixon SJ, Lemberg KM, Lamprecht MR, Skouta R, Zaitsev EM, Gleason CE et al. Ferroptosis: an iron-dependent form of nonapoptotic cell death. Cell 2012; 149: 1060-1072.

21. Dixon SJ, Patel DN, Welsch M, Skouta R, Lee ED, Hayano M et al. Pharmacological inhibition of cystine-glutamate exchange induces endoplasmic reticulum stress and ferroptosis. Elife 2014; 3: e02523.

22. Yang WS, SriRamaratnam R, Welsch ME, Shimada K, Skouta R, Viswanathan VS et al. Regulation of ferroptotic cancer cell death by GPX4. Cell 2014; 156: 317-331.

23. Massi P, Vaccani A, Ceruti S, Colombo A, Abbracchio MP, Parolaro D. Antitumor effects of cannabidiol, a nonpsychoactive cannabinoid, on human glioma cell lines. J Pharmacol Exp Ther 2004; 308: 838-845.

24. Massi P, Valenti M, Vaccani A, Gasperi V, Perletti G, Marras E et al. 5-Lipoxygenase and anandamide hydrolase (FAAH) mediate the antitumor activity of cannabidiol, a non-psychoactive cannabinoid. J Neurochem 2008; 104: 1091-1100.

25. De Petrocellis L, Ligresti A, Schiano Moriello A, lappelli M, Verde R, Stott CG et al. Non-THC cannabinoids inhibit prostate carcinoma growth in vitro and in vivo: pro-apoptotic effects and underlying mechanisms. Br J Pharmacol 2012; 168: 79-102.

26. McKallip RJ, Jia W, Schlomer J, Warren JW, Nagarkatti PS, Nagarkatti M. Cannabidiolinduced apoptosis in human leukemia cells: a novel role of cannabidiol in the regulation of p22phox and Nox4 expression. Mol Pharmacol 2006; 70: 897-908.

27. Murase R, Kawamura R, Singer E, Pakdel A, Sarma P, Judkins J et al. Targeting multiple cannabinoid anti-tumour pathways with a resorcinol derivative leads to inhibition of advanced stages of breast cancer. Br J Pharmacol 2014; 171: 4464-4477.

28. Ramer R, Bublitz K, Freimuth N, Merkord J, Rohde H, Haustein M et al. Cannabidiol inhibits lung cancer cell invasion and metastasis via intercellular adhesion molecule-1. FASEB $J$ 2012; 26: 1535-1548.

29. Sato A, Okada M, Shibuya K, Watanabe E, Seino S, Narita Y et al. Pivotal role for ROS activation of p38 MAPK in the control of differentiation and tumor-initiating capacity of gliomainitiating cells. Stem Cell Res 2014; 12: 119-131.

30. Hegi ME, Diserens AC, Gorlia T, Hamou MF, de Tribolet N, Weller M et al. MGMT gene silencing and benefit from temozolomide in glioblastoma. N Engl J Med 2005; 352 997-1003.

31. Savaskan NE, Eyupoglu IY. xCT modulation in gliomas: relevance to energy metabolism and tumor microenvironment normalization. Ann Anat 2010; 192: 309-313.

32. Ishimoto T, Nagano O, Yae T, Tamada M, Motohara T, Oshima $\mathrm{H}$ et al. CD44 variant regulates redox status in cancer cells by stabilizing the $\mathrm{xCT}$ subunit of system $\mathrm{xc}(-)$ and thereby promotes tumor growth. Cancer Cell 2011; 19: 387-400.

33. Timmerman LA, Holton T, Yuneva M, Louie RJ, Padro M, Daemen A et al. Glutamine sensitivity analysis identifies the $\mathrm{xCT}$ antiporter as a common triple-negative breast tumor therapeutic target. Cancer Cell 2013; 24: 450-465.

34. Dixon SJ, Stockwell BR. The role of iron and reactive oxygen species in cell death. Nat Chem Biol 2013; 10: 9-17

(1) Cell Death and Disease is an open-access journal published by Nature Publishing Group. This work is licensed under a Creative Commons Attribution 4.0 International Licence. The images or other third party material in this article are included in the article's Creative Commons licence, unless indicated otherwise in the credit line; if the material is not included under the Creative Commons licence, users will need to obtain permission from the licence holder to reproduce the material. To view a copy of this licence, visit http://creativecommons.org/licenses/by/4.0 